\title{
Hipotensão Pós-exercício Aeróbio: Uma Revisão Sistemática
}

\author{
Post-exercise Hypotension: a Systematic Review
}

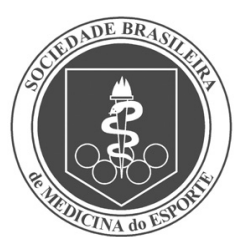

Artigo de Revisão
Juliano Casonatto',

Marcos Doederlein Polito

1. Programa de Pós-Gradução Stricto Sensu UEM/UEL.

\section{Endereço para correspondência:}

Marcos Polito - Universidade

Estadual de Londrina - Centro

de Educação Física e Esporte

- Departamento de Educação Física - Rodovia Celso Garcia Cid, km 380

- Campus Universitário - 86051-901

-- Londrina, PR.- Tel.: (43) 3371-4238.

E-mail: marcospolito@uel.br

Submetido em 04/09/2008 Versão final recebida em 30/10/2008 Aceito em 28/11/2008

\section{RESUMO}

Diversos estudos investigaram os efeitos hipotensores após uma sessão de exercício aeróbio em humanos. No entanto, vários aspectos permanecem obscuros em relação à hipotensão pós-exercício (HPE), uma vez que diversas variáveis podem influenciar a resposta hipotensora, como intensidade, duração, tipo de exercício, estado clínico, faixa etária, etnia, sexo e estado de treinamento. Nesse sentido, o objetivo do presente estudo foi revisar sistematicamente a literatura, relacionando as principais variáveis da prescrição de uma sessão de exercício aeróbio e a HPE, assim como apresentar os possíveis mecanismos envolvidos. Foram encontrados 55 estudos que abrangeram a temática HPE e exercício aeróbio em humanos. A ocorrência da HPE está bem estabelecida na literatura, já que vários estudos identificaram reduções da pressão arterial em normotensos e hipertensos. Porém, os possíveis moduladores das respostas hipotensoras, como intensidade e duração da sessão de exercício, ainda são contraditórios. Em relação ao tipo de exercício, porém, existem indicativos de que os realizados de forma intermitente e que utilizam maior massa muscular podem acarretar maior HPE. Além disso, hipertensos devem apresentar maior magnitude e duração da HPE. Contudo, existem lacunas em relação aos diversos mecanismos fisiológicos envolvidos, que parecem ser diferentes entre normotensos e hipertensos.

Palavras-chave: pressão arterial, exercício aeróbio, fisiologia cardiovascular.

\section{ABSTRACT}

Several studies have investigated the hypotensor effects after an aerobic exercise session in humans. However, many aspects remain unclear concerning post-exercise hypotension (PEH), once many variables can influence on the hypotensor response, such as intensity, duration, type of exercise, clinical status, age, ethnic group, sex and training level. Therefore, the aim of the present study was to systematically review the literature, relating the main prescription variables of an aerobic exercise session to $\mathrm{PEH}$, as well as to present the possible mechanisms involved. Fifty-five studies which approached PEH and aerobic exercise in humans have been found. PEH occurrence is well-established in the literature, once many studies have identified reduction in blood pressure in normotensive and hypertensive individuals. Nevertheless, the possible modulators of the hypotensive responses, such as intensity and duration of the exercise session, are contradictory yet. Concerning the type of exercise, there are indications that intermittent exercise which uses greater muscular mass may lead to higher PEH. Additionally, hypertensive patients should present greater magnitude and PEH duration. However, there are some gaps regarding the several physiological mechanisms involved, which seem to be different between normotensive and hypertensive individuals.

Keywords: blood pressure, aerobic exercise, cardiovascular physiology.

\section{INTRODUÇÃO}

A hipertensão arterial é um importante problema de saúde pública em países desenvolvidos e em desenvolvimento(1). Por isso, são adotadas estratégias preventivas quanto ao aumento da pressão arterial (PA) de repouso. Dentro de tais estratégias, a prática regular de exercícios físicos é uma intervenção pouco onerosa e não farmacológica, possibilitando reduções significativas na PA de repouso(2).

A redução na PA de repouso pelo exercício pode ocorrer de forma crônica ou aguda. A redução crônica provém do treinamento sistematizado, tendo o modelo aeróbio como um dos mais eficientes ${ }^{(2,3)}$. Já a redução aguda ocorre nos minutos ou horas subsequentes à prática, por meio do efeito denominado hipotensão pós-exercício (HPE) ${ }^{(2)}$. A HPE possui elevada significância clínica, principalmente em hipertensos, pois pode atuar como hipotensor não farmacológico. Embora vários estudos tenham investigado a HPE, ainda existem certas dúvidas quanto às principais variáveis da prescrição relacionadas a esste efeito. Por exemplo, em um estudo publicado recentemente ${ }^{(4)}$, os autores demonstraram que a HPE é mais prolongada após uma sessão de exercício aeróbio com maior duração (30 min vs 50 min), enquanto outro experimento ${ }^{(5)}$ verificou que a HPE se manifesta de maneira similar, independentemente da duração do exercício.

Dessa forma, o objetivo do presente estudo foi revisar sistematicamente a literatura sobre o exercício aeróbio e a HPE, buscando relação entre as principais variáveis da prescrição.

\section{PROCEDIMENTOS PARA A BUSCA DE ARTIGOS}

Foi utilizado o modelo sistemático de pesquisa na base de dados Medline sem limites de data até de julho de 2008. Para tanto, foram utilizados os termos: post-exercise, postexercise, hypotension, dynamic exercise, aerobic exercise, blood pressure e acute blood pressure response, de forma isolada e combinada em citações no título ou resumo. Foram considerados apenas os artigos publicados no idioma inglês, que acompanharam o comportamento da PA após o exercício aeróbio por no mínimo 20 min e foram realizados em humanos. Foram localizados 
98 estudos. Desses, 22 abordaram o efeito crônico do exercício, 10 utilizaram modelos animais, oito investigaram hipotensão ortostática, quatro acompanharam a PA pós-exercício por tempo inferior a 20 min e um estudo foi de caso. Dessa forma, 53 artigos atenderam aos critérios para compor as referências do presente estudo.

A tabela 1 ilustra todos os estudos que compuseram a presente revisão. Considerando a grande quantidade de dados, a tabela 2 resume os principais tópicos relacionados à prescrição do exercício.

\section{A HIPOTENSÃO PÓS-EXERCÍCIO}

Um dos primeiros relatos na literatura sobre a ocorrência do fenômeno da HPE foi descrito há mais de 100 anos por Leonard Hill(6), quando acompanhou por 90 min a PA de um homem após uma corrida de 400 jardas. Porém, somente a partir da década de 1980 iniciou-se sistematicamente a investigação desse fenômeno, relacionando-o como efeito clínico relevante ${ }^{(7)}$. A partir daí, a literatura produzida confirma a HPE em sujeitos hipertensos e pré-hipertensos ${ }^{(7-19)}$. No entanto, a ocorrência desse fenômeno em humanos normotensos ainda permanece inconsistente. Apesar disso, alguns estudos identificaram a HPE em normotensos, porém com menor magnitude que em hipertensos ${ }^{(20,21)}$. Essa diferença entre hipertensos e normotensos pode estar atrelada à forma de prescrição do exercício e aos possíveis mecanismos fisiológicos envolvidos na HPE. As próximas sessões propõem-se a discutir os aspectos mais relevantes destes tópicos.

Tabela 1. Comportamento da pressão arterial após uma sessão de exercício aeróbio

\begin{tabular}{|c|c|c|c|c|c|c|c|c|}
\hline Estudo & Amostra & Sexo & $\mathrm{N}$ & Exerćício & Intensidade & Duração & $\begin{array}{c}\text { Monitorização } \\
\text { pós-exercício }\end{array}$ & Efeito pós-exercício \\
\hline \multicolumn{9}{|l|}{ Normotensos } \\
\hline Moraes et al..$^{51}$ & Sedentários (38 \pm 4 anos) & M & 8 & Cicloergômetro & $70 \% \mathrm{FC}_{\text {reserva }}$ & $35 \mathrm{~min}$. & $60 \mathrm{~min}$. & $\downarrow$ PAS aos 30,45 e $60 \mathrm{~min} / \downarrow$ PAD aos $45 \mathrm{~min}$ \\
\hline Pescatello et al? & Sedentários (41 \pm 2 anos) & M & 6 & Cicloergômetro & 40 e $70 \% \mathrm{VO}_{2 \max }$ & $30 \mathrm{~min}$. & $13 \mathrm{~h}$. & $\downarrow$ PAS nos momentos 1, 2, 6, 7 e $12 \mathrm{~h} /$ PAD NS \\
\hline Forjaz et al. ${ }^{21}$ & \begin{tabular}{|l} 
Sedentários (33 \pm 2 anos) \\
\end{tabular} & $\mathrm{M} / \mathrm{F}$ & 30 & Cicloergômetro & $50 \% \mathrm{VO}_{2 \text { pico }}$ & $45 \mathrm{~min}$. & $24 \mathrm{~h}$. & $\downarrow$ PAS e PAD (média 24h) \\
\hline MacDonald et al. ${ }^{85}$ & Sedentários (24 \pm 2 anos) & M & 13 & Cicloergômetro & $65 \% \mathrm{VO}_{2 \text { máx }}$ & $15 \mathrm{~min}$. & $60 \mathrm{~min}$. & $\downarrow$ PAS entre 10-60 min / PAD NS \\
\hline MacDonald et al..$^{34}$ & Sedentários (35 \pm 16 anos) & $M / F$ & 10 & Cicloergômetro & 50 e $75 \% \mathrm{VO}_{2 \text { pico }}$ & $30 \mathrm{~min}$. & $60 \mathrm{~min}$ & 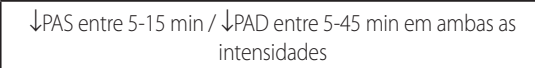 \\
\hline Forjaz et al. ${ }^{29}$ & Sedentários (22 \pm 1 anos) & $M / F$ & 10 & Cicloergômetro & $50 \% \mathrm{VO}_{\text {2pico }}$ & $25 \mathrm{~min}$. & $90 \mathrm{~min}$. & $\downarrow$ PAS entre $45-75 \mathrm{~min} / \downarrow$ PAD entre $15-90 \mathrm{~min}$ \\
\hline Forjaz et al. ${ }^{29}$ & Sedentários (22 \pm 1 anos) & $\mathrm{M} / \mathrm{F}$ & 10 & Cicloergômetro & $50 \% \mathrm{VO}_{2 \text { pico }}$ & $45 \mathrm{~min}$. & $90 \mathrm{~min}$. & $\downarrow$ PAS entre 30-90 min / \PAD entre $15-90 \mathrm{~min}$ \\
\hline Forjaz et al. ${ }^{33}$ & Sedentários (22 \pm 1 anos) & $\mathrm{M} / \mathrm{F}$ & 12 & Cicloergômetro & 30,50 e $80 \% \mathrm{VO}_{\text {2pico }}$ & $45 \mathrm{~min}$. & $90 \mathrm{~min}$. & \PAS e PAD entre $30-90$ min em todas as intensidades. \\
\hline Headley et $a l^{6.6}$ & Sedentários (25 \pm 1 anos) & M & 19 & Esteira ergométrica & $50-60 \% F C_{\text {reserva }}$ & $40 \mathrm{~min}$. & $120 \mathrm{~min}$. & $\downarrow$ PAS entre 30-120 min / PAD NS \\
\hline Harvey et a $l^{52}$ & 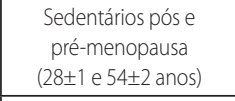 & $\mathrm{F}$ & 27 & Esteira ergométrica & $60 \% \mathrm{VO}_{2 \text { máx }}$ & $45 \mathrm{~min}$. & $90 \mathrm{~min}$. & $\begin{array}{l}\text { \PAS e PAD na amosra pós-menopausa (média } 90 \text { min) / } \\
\text { Pré-menopausa NS }\end{array}$ \\
\hline Birch et al. ${ }^{86}$ & Ativos (20 \pm 1 anos) & $\mathrm{F}$ & 15 & Cicloergômetro & $60 \% \mathrm{VO}_{2 \text { máx }}$ & $30 \mathrm{~min}$. & $60 \mathrm{~min}$. & $\downarrow$ PAS e PAD aos 5 e $15 \mathrm{~min}$ \\
\hline Terziotti et $1 . .^{87}$ & Sedentários (24-38 anos) & M & 12 & Cicloergômetro & 50 e $80 \%$ do LAl & $20 \mathrm{~min}$. & $180 \mathrm{~min}$. & \PAS aos 25 min em ambas as intensidades / PAD NS \\
\hline MacDonald et al. ${ }^{30}$ & Sedentários (22 \pm 1 anos) & M & 13 & Cicloergômetro & $70 \% \mathrm{VO}_{2 \text { pico }}$ & 15,30 e $45 \mathrm{~min}$. & $60 \mathrm{~min}$ & $\begin{array}{c}\text { \PAS entre 5-60 min / } \downarrow \text { PAD entre } 30-45 \text { min nos três modelos } \\
\text { de exercício }\end{array}$ \\
\hline Jones et al. ${ }^{4}$ & Ativos (28 \pm 6 anos) & M & 7 & Cicloergômetro & 70 e $40 \% \mathrm{VO}_{2 \text { pico }}$ & $30 \mathrm{~min}$ e $\approx 50 \mathrm{~min}$ & $20 \mathrm{~min}$. & $\downarrow$ PAS aos $20 \mathrm{~min} /$ PAD NS \\
\hline Blanchard et al. ${ }^{1 * *}$ & Sedentários (44 \pm 1 anos) & M & 47 & Cicloergômetro & 40 e $60 \% \mathrm{VO}_{2 \operatorname{máx}}$ & $40 \mathrm{~min}$. & $14 \mathrm{~h}$. & \PAS e PAD no exercício de $40 \%$ do $\mathrm{VO}_{2 \text { máx }}$ (média $14 \mathrm{~h}$ ) \\
\hline Pescatello et $a l_{.}^{8}$ & Sedentários (34 \pm 2 anos) & $\mathrm{F}$ & 11 & Cicloergômetro & $60 \% \mathrm{VO}_{2 \text { máx }}$ & $30 \mathrm{~min}$. & $24 \mathrm{~h}$. & NS \\
\hline Wallace et al. ${ }^{9}$ & Sedentários (50 \pm 11 anos) & $\mathrm{M} / \mathrm{F}$ & 25 & Esteira ergométrica & $50 \% \mathrm{VO}_{2 \operatorname{máx}}$ & $50 \mathrm{~min}$. & $24 \mathrm{~h}$. & NS (média 24h, sono e vigília) \\
\hline Bermudes et al..$^{20}$ & Sedentários (40-50 anos) & M & 25 & Cicloergômetro & $60-80 \% F C_{\text {máx }}$ & $45 \mathrm{~min}$. & $24 \mathrm{~h}$. & $\downarrow$ PAD (média 24h) / PAS NS \\
\hline Halliwill et al. ${ }^{71}$ & Sedentários (22-27 anos) & $\mathrm{M} / \mathrm{F}$ & 9 & Cicloergômetro & $60 \% \mathrm{VO}_{2 \text { pico }}$ & $60 \mathrm{~min}$. & $60 \mathrm{~min}$. & \PAS / PAD NS (média 60 min) \\
\hline Lockwood et al. ${ }^{26}$ & Sedentários (20-32 anos) & M & 11 & Cicloergômetro & $60 \% \mathrm{VO}_{2 \text { pico }}$ & $60 \mathrm{~min}$. & $90 \mathrm{~min}$. & $\downarrow$ PAM aos 30 e $60 \mathrm{~min}$ \\
\hline Halliwill et al.23 & Sedentários (21-28 anos) & M & 12 & Cicloergômetro & $60 \% \mathrm{VO}_{2 \text { pico }}$ & $60 \mathrm{~min}$. & $165 \mathrm{~min}$. & $\downarrow$ PAM aos 30 e $75 \mathrm{~min}$ \\
\hline Wallace et al. ${ }^{28}$ & 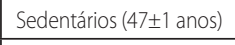 & $\mathrm{M} / \mathrm{F}$ & 36 & Esteira ergométrica & $50 \% \mathrm{VO}_{2 \text { máx }}$ & $50 \mathrm{~min}$. & $24 \mathrm{~h}$. & NS (média 24h) \\
\hline Forjaz et al. ${ }^{35}$ & Sedentários (24 \pm 1 anos) & $\mathrm{M} / \mathrm{F}$ & 23 & Cicloergômetro & 30,50 e $75 \% \mathrm{VO}_{2 \text { pico }}$ & $45 \mathrm{~min}$. & $90 \mathrm{~min}$. & $\downarrow$ PAS e PAD nas intensidades $50 \%$ e $75 \%$ do $V_{2} O_{2 p i c o}$ \\
\hline Alderman et al.36 & Ativos (18-35 anos) & $M / F$ & 90 & Esteira ergométrica & $\begin{array}{l}70-85 \% \text { e } 50-55 \% \\
\mathrm{VO}_{2 \operatorname{máx}}\end{array}$ & $30 \mathrm{~min}$. & $60 \mathrm{~min}$. & $\downarrow$ PAS aos 5,30 e $60 \mathrm{~min} / \downarrow$ PAD aos 5 e $30 \mathrm{~min}$ \\
\hline Piepoli et al.37 & Sedentários (24-34 anos) & $M / F$ & 8 & Cicloergômetro & $\begin{array}{l}\text { Exercício máximo, } \\
\text { moderado e leve }\end{array}$ & - & $60 \mathrm{~min}$. & $\begin{array}{c}\downarrow \text { PAS aos } 5 \mathrm{~min} / \downarrow \text { PAD aos } 5,10,45 \text { e } 60 \text { min após o exercício } \\
\text { máximo }\end{array}$ \\
\hline Raglin et al. ${ }^{67}$ & Atletas (Adultos) & $M / F$ & 26 & Cicloergômetro & $70-80 \%$ da CMl & $30 \mathrm{~min}$. & $60 \mathrm{~min}$. & $\downarrow$ PAS / PAD NS \\
\hline Coats et al. ${ }^{54}$ & Sedentários (17-47 anos) & $\mathrm{M} / \mathrm{F}$ & 13 & Cicloergômetro & Teste máximo & - & $60 \mathrm{~min}$. & $\downarrow$ PAS após $45 \mathrm{~min} / \downarrow$ PAD durante todo o período \\
\hline Brown et al. ${ }^{68}$ & Sedentários (meiadade) & $\mathrm{M} / \mathrm{F}$ & 7 & Cicloergômetro & $70 \% \mathrm{FC}_{\text {reserva }}$ & $25 \mathrm{~min}$. & $60 \mathrm{~min}$. & $\downarrow$ PAS após $5 \mathrm{~min} / \downarrow$ PAD após $15 \mathrm{~min}$ \\
\hline Pescatello et al. ${ }^{14 *}$ & Sedentários (18-55 anos) & $\mathrm{M}$ & 49 & Cicloergômetro & 40 e $60 \% \mathrm{VO}_{2 \text { máx }}$ & $30 \mathrm{~min}$. & $9 \mathrm{~h}$. & $\downarrow$ PAD e $\uparrow$ PAS (média 9 h.). \\
\hline Dujic et al. ${ }^{75}$ & Ativos (22 \pm 3 anos) & M & 20 & Pista de atletismo & Exercício máximo & - & $60 \mathrm{~min}$. & $\downarrow$ PAS e PAD em todo período \\
\hline Jones et al. ${ }^{31}$ & Ativos (26 \pm 5 anos) & M & 12 & Cicloergômetro & $70 \% \mathrm{VO}_{\text {2pico }}$ & $30 \mathrm{~min}$. & $20 \mathrm{~min}$. & $\downarrow$ PAS em todo período / $\downarrow$ PAD aos 5 min \\
\hline Rondon et al. ${ }^{88}$ & Sedentários (68 \pm 1 anos) & $\mathrm{M} / \mathrm{F}$ & 18 & Cicloergômetro & $50 \% \mathrm{VO}_{2 \text { pico }}$ & $45 \mathrm{~min}$. & $90 \mathrm{~min}$. & NS \\
\hline Pescatello et al. ${ }^{12 \dagger}$ & Sedentários (19-45 anos) & $\mathrm{F}$ & 11 & Cicloergômetro & $60 \% \mathrm{VO}_{2 \operatorname{máx}}$ & $30 \mathrm{~min}$. & $\approx 24 \mathrm{~h}$. & NS (média aproximada de 24h) \\
\hline Pescatello et al. ${ }^{12 \neq}$ & Sedentários (19-45 anos) & $\mathrm{F}$ & 10 & Cicloergômetro & $60 \% \mathrm{VO}_{2 \max }$ & $30 \mathrm{~min}$. & $\approx 24 \mathrm{~h}$. & \PAD / PAS NS (média aproximada de 24h) \\
\hline Piepoli et a.$^{81}$ & Sedentários (adultos) & $\mathrm{M} / \mathrm{F}$ & 10 & Cicloergômetro & Exercício máximo & - & $60 \mathrm{~min}$. & $\downarrow$ PAD em todo período \\
\hline Brownley et al.22 & Sedentários (adultos) & $\mathrm{M} / \mathrm{F}$ & 20 & Cicloergômetro & Moderada & $20 \mathrm{~min}$. & $48 \mathrm{~h}$. & NS \\
\hline Isea et al..$^{5}$ & Sedentários (adultos) & $M$ & 6 & Cicloergômetro & Exercício máximo & - & $4 \mathrm{~h}$. & \PAS e PAD nas primeiras três horas \\
\hline Cleroux et al. ${ }^{56}$ & Sedentários (41 \pm 2 anos) & $\mathrm{M} / \mathrm{F}$ & 9 & Cicloergômetro & $50 \% \mathrm{VO}_{\text {2pico }}$ & $30 \mathrm{~min}$. & $90 \mathrm{~min}$. & NS \\
\hline Kaufman et al. .99 & Sedentários (19-29 anos) & M & 8 & Esteira ergométrica & $67 \% \mathrm{FC}_{\text {máx }}$ & $50 \mathrm{~min}$. & $60 \mathrm{~min}$. & $\downarrow P A S$ e PAD \\
\hline Kaufman et al..$^{69}$ & Sedentários (35-62 anos) & M & 8 & Esteira ergométrica & $67 \% \mathrm{FC}_{\text {max }}$ & $50 \mathrm{~min}$. & $60 \mathrm{~min}$. & $\downarrow P A S$ e PAD \\
\hline
\end{tabular}




\begin{tabular}{|c|c|c|c|c|c|c|c|c|}
\hline Estudo & Amostra & Sexo & $\mathrm{N}$ & Exercício & Intensidade & Duração & $\begin{array}{c}\text { Monitorização } \\
\text { pós-exercício }\end{array}$ & Efeito pós-exercício \\
\hline Senitko et al. ${ }^{74}$ & Sedentários (25 \pm 5 anos) & $\mathrm{M} / \mathrm{F}$ & 16 & Cicloergômetro & $60 \% \mathrm{VO}_{2 \text { pico }}$ & $60 \mathrm{~min}$ & $60 \mathrm{~min}$. & $\downarrow P A M$ aos 30 e $60 \mathrm{~min}$ \\
\hline Senitko et al. ${ }^{74}$ & Ativos (27 \pm 4 anos) & $M / F$ & 16 & Cicloergômetro & $60 \% \mathrm{VO}_{2 \text { pico }}$ & $60 \mathrm{~min}$ & $60 \mathrm{~min}$. & $\downarrow P A M$ aos 30 e $60 \mathrm{~min}$ \\
\hline
\end{tabular}

\begin{tabular}{|c|c|c|c|c|c|}
\hline \multicolumn{6}{|l|}{ PA elevada } \\
\hline Syme et al. ${ }^{16}$ & 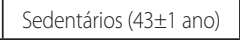 & $M$ & 50 & Cicloergômetro & 40 e $60 \% \mathrm{VO}_{2 \text { máx }}$ \\
\hline Guidry et al..$^{5}$ & Sedentários (43 \pm 2 anos) & $M$ & 45 & Cicloergômetro & 40 e $60 \% \mathrm{VO}_{2 \text { máx }}$ \\
\hline Brownley et al.22 & Sedentários (adultos) & $\mathrm{M} / \mathrm{F}$ & 11 & Cicloergômetro & Moderada \\
\hline Pescatello et al. ${ }^{19}$ & 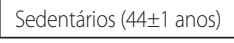 & M & 50 & Cicloergômetro & 40 e $60 \% \mathrm{VO}_{2 \text { máx }}$ \\
\hline \multicolumn{6}{|l|}{ Pré-hipertensos } \\
\hline MacDonald et al..$^{32}$ & Sedentários ( $23 \pm 4$ anos) & $M / F$ & 8 & Cicloergômetro & $70 \% \mathrm{VO}_{2 \text { pico }}$ \\
\hline MacDonald et al..$^{30}$ & Sedentários (23 \pm 4 anos) & $M / F$ & 8 & Cicloergômetro & $70 \% \mathrm{VO}_{2 \text { pico }}$ \\
\hline MacDonald et al..$^{45}$ & Sedentários (23 \pm 4 anos) & $M / F$ & 9 & $\begin{array}{c}\text { Ergômetro de Braço } \\
\text { e Cicloergômetro }\end{array}$ & $\begin{array}{l}65 \% \mathrm{VO}_{2 \text { pico }} \text { (braço } \\
70 \% \mathrm{VO}_{2 \text { pico }} \text { (pern }\end{array}$ \\
\hline MacDonald et al. ${ }^{27}$ & Sedentários ( $25 \pm 5$ anos) & $M / F$ & 11 & Cicloergômetro & $70 \% \mathrm{VO}_{2 \text { pico }}$ \\
\hline Park et al. ${ }^{18}$ & 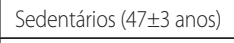 & $M / F$ & 21 & Caminhada & $50 \% V_{2 \text { pico }}$ \\
\hline Pescatello et al. ${ }^{12 \dagger}$ & Sedentários (19-45 anos) & $\mathrm{F}$ & 7 & Cicloergômetro & $50 \% \mathrm{VO}_{2 \text { máx }}$ \\
\hline Pescatello et al. ${ }^{12 \neq}$ & Sedentários (19-45 anos) & $\mathrm{F}$ & 5 & Cicloergômetro & $50 \% \mathrm{VO}_{2 \operatorname{máx}}$ \\
\hline Headley et al..$^{2}$ & Sedentários (meia idade) & $\mathrm{F}$ & 20 & Esteira ergométrica & Moderada \\
\hline \multicolumn{6}{|l|}{ Hipertensos } \\
\hline Blanchard et al..$^{17 *}$ & 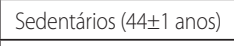 & $M$ & 47 & Cicloergômetro & 40 e $60 \% \mathrm{VO}_{2 \text { máx }}$ \\
\hline Pescatello et al? & \begin{tabular}{|l|} 
Sedentários ( $44 \pm 4$ anos) \\
\end{tabular} & M & 6 & Cicloergômetro & 40 e $70 \% \mathrm{VO}_{2 \text { máx }}$ \\
\hline Moraes et al.51 & Sedentários (38 \pm 4 anos) & M & 10 & Cicloergômetro & $70 \% \mathrm{FC}_{\text {reserva }}$ \\
\hline Rueckert et al. ${ }^{43}$ & Sedentários (50 \pm 2 anos) & $M / F$ & 18 & Esteira ergométrica & $70 \% \mathrm{FC}_{\text {reserva }}$ \\
\hline Forjaz et a $l^{2.1}$ & Sedentários (36 2 anos) & $M / F$ & 23 & Cicloergômetro & $50 \% \mathrm{VO}_{2 \text { pico }}$ \\
\hline Taylor-Tolbert et a!. ${ }^{13}$ & Sedentários (60 \pm 6 anos) & $\mathrm{M}$ & 11 & Esteira ergométrica & $70 \% \mathrm{VO}_{2 \max }$ \\
\hline Pescatello et $a l^{8}$ & Sedentários (38 \pm 2 anos) & $\mathrm{F}$ & 7 & Cicloergômetro & $60 \% \mathrm{VO}_{2 \text { max }}$ \\
\hline Wallace et al. ${ }^{9}$ & Sedentários (48 \pm 11 anos) & $M / F$ & 21 & Esteira ergométrica & $50 \% \mathrm{VO}_{2 \max }$ \\
\hline Pontes et al. ${ }^{46}$ & Sedentários (adultos) & $M / F$ & 16 & Corrida aquática & $50 \% \mathrm{VO}_{2 \text { pico }}$ \\
\hline Quinn. $^{10}$ & Sedentários (42 \pm 8 anos) & $M / F$ & 16 & Esteira ergométrica & 50 e $75 \% \mathrm{VO}_{2 \text { máx }}$ \\
\hline Wallace et al. ${ }^{28}$ & Sedentários (48 \pm 13 anos) & $M / F$ & 25 & Esteira ergométrica & $50 \% \mathrm{VO}_{2 \operatorname{máx} .}$ \\
\hline Hagberg et al..$^{50}$ & Sedentários (60-69 anos) & $\mathrm{M} / \mathrm{F}$ & 24 & Esteira ergométrica & 50 e $70 \% \mathrm{VO}_{2 \text { máx }}$ \\
\hline Rondon et al. ${ }^{88}$ & Sedentários (69 \pm 2 anos) & $M / F$ & 24 & Cicloergômetro & $50 \% \mathrm{VO}_{2 \text { máx. }}$ \\
\hline Cleroux et al.56 & Sedentários (44 \pm 2 anos) & $M / F$ & 13 & Cicloergômetro & $50 \% \mathrm{VO}_{2 \text { pico }}$ \\
\hline Kaufman et al..$^{69}$ & Sedentários (44-57 anos) & M & 8 & Esteira ergométrica & $67 \% \mathrm{FC}_{\text {máx }}$ \\
\hline Ciolac et al. ${ }^{84}$ & Sedentários (?) & $?$ & 52 & Cicloergômetro & $50-80 \% \mathrm{FC}_{\text {reserva }}$ \\
\hline Bennett et al. ${ }^{39}$ & Sedentários (31-62 anos) & M & 7 & Esteira ergométrica & Exercício Intermiter \\
\hline $\begin{array}{l}\text { *Amostra de normot } \\
\text { acionalmente ativos; } \\
\text { individual; } \mathrm{VO}_{2 \text { máx }}=\mathrm{V} \\
\text { Pressão arterial médi }\end{array}$ & a; $\downarrow$ redução significativ & 1. & & $\begin{array}{l}\text { e indivíduos branc } \\
\text { gular e sistematizac } \\
\text { me de oxigênio pi } \\
\text { ficativo; NS= Não s }\end{array}$ & $\begin{array}{l}\mathrm{cos} ; \neq=\text { Somente } \\
\text { da/Atletas profissio } \\
\text { ico; Fc } c_{\text {reserva }}=\text { Frequ } \\
\text { significante. }\end{array}$ \\
\hline & Mínimo & & & & édia (DP) \\
\hline
\end{tabular}

\section{Normotensos}

Intensidade

\begin{tabular}{l|l|l|l}
\hline $\mathrm{VO}_{2 \text { máx }}(\%)$ & 40 & 85 & $57,5(11,5)$ \\
\hline $\mathrm{VO}_{\text {2pico }}(\%)$ & 30 & 80 & $56,3(13,5)$ \\
\hline $\mathrm{FC}_{\text {reserva }}(\%)$ & 50 & 80 & $65,0(10,4)$ \\
\hline Duração (min) & 15 & 60 & $38,6(12,9)$ \\
\hline
\end{tabular}

\section{Hipertensos*}

$\underline{\text { Intensidade }}$

\begin{tabular}{l|l|l|l}
\hline $\mathrm{VO}_{2 \text { máx }}(\%)$ & 40 & 75 & $54,7(11,4)$ \\
\hline $\mathrm{VO}_{2 \text { pico }}(\%)$ & 50 & 70 & $61,8(9,9)$ \\
\hline $\mathrm{FC}_{\text {reserva }}(\%)$ & 50 & 80 & $66(11,4)$ \\
\hline Duração (min) & 10 & 50 & $34,8(10,3)$ \\
\hline
\end{tabular}

*Indivíduos classificados como: portadores de pressão arterial elevada, pré-hipertensos e hipertensos. $\mathrm{DP}=$ Desvio padrão; $\mathrm{VO}_{2 \max }=$ Volume máximo de oxigênio; $\mathrm{VO}_{2 \text { pico }}=$ Volume de oxigênio pico; $\mathrm{FC}_{\text {reserva }}=$ Frequência cardíaca de reserva.

\section{A INFLUÊNCIA DA INTENSIDADE DO EXERCÍCIO}

Não é verificado consenso em relação à intensidade do exercício aeróbio sobre a magnitude e duração da HPE. A maioria dos estudos que analisou a PA após a prática de exercícios aeróbios utilizou protocolos em cicloergômetro ou esteira ergométrica, com intensidade entre 40 e100\% da capacidade máxima, monitorada pelo volume máximo de oxigênio, frequência cardíaca de reserva ou frequência cardíaca máxima prevista(22-28). Assim, estudos que empregaram intensidades relativamente baixas ${ }^{(18,21,29)}$ ou que utilizaram intensidades elevadas ${ }^{(30-32),}$ demonstraram HPE em normotensos ${ }^{(21,29-31)}$ e em hipertensos ${ }^{(18,21,30,32)}$. Além disso, estudos que realizaram comparações diretas da intensidade do exercício indicam que a HPE pode ocorrer independentemente da intensidade em normotensos ${ }^{(4,33-37)}$ e hipertensos ${ }^{(14)}$.

Por outro lado, os resultados das investigações são conflitantes quanto à magnitude e duração da HPE em função da intensidade. Alguns experimentos mostraram que exercícios aeróbios de intensidade elevada produzem maior magnitude ${ }^{(4,35-37)}$ e duração( ${ }^{(35,37)}$ da HPE quando comparados com os exercícios de intensidade moderada. Porém, outras investigações não encontraram diferenças na magnitude e na duração da HPE geradas pela intensidade em sujeitos normotensos ${ }^{(33,34)}$ e hipertensos ${ }^{(14)}$. 
Em estudo publicado recentemente, Jones et al. ${ }^{(4)}$ compararam o efeito da intensidade do exercício sobre a HPE controlando o volume total de trabalho. Os autores verificaram diferenças significativas na magnitude da HPE entre a prática de exercício intenso e moderado. Além disso, após 20 min de acompanhamento, o exercício intenso demonstrou causar redução da resistência periférica total e aumento da condutância vascular cutânea. Dessa forma, pode-se supor que a intensidade do exercício influencie de alguma forma o comportamento da HPE. No entanto, não é adequado para sujeitos hipertensos realizar o exercício em intensidades elevadas, reduzindo o poder de discussão quando relacionado com a validade externa.

\section{A INFLUÊNCIA DA DURAÇÃO DO EXERCÍCIO}

A HPE tem sido observada após exercícios de curta ${ }^{(30)}$ (10 min) e longa duração ${ }^{(38)}$ (170 min). No entanto, a maioria dos estudos utilizou protocolos com duração entre 20 e 60 min. Esses experimentos apresentam metodologias distintas em relação ao tipo e intensidade dos exercícios, estado clínico, nível de treinamento físico, idade, sexo e raça da amostra. Essas incompatibilidades comprometem comparações interexperimentos. Porém, algumas pesquisas comparam diferentes durações do esforço sobre a HPE em humanos com PA normal(4,29,30,39) e elevada ${ }^{(5,30,39,40)}$. Alguns pesquisadores também estudaram o efeito da duração do exercício em sujeitos fisicamente ativos ${ }^{(4)}$ e em cobaias $^{(41)}$. Analisando tais estudos, foram identificados experimentos que demonstram que uma sessão de exercícios com maior duração potencializa tanto a magnitude ${ }^{(4,29,39-41)}$ quanto a duração(4,29,40,41) da HPE. Tais resultados, em geral, são atribuídos ao aumento das respostas neurais e hormonais decorrentes da exposição ao estresse fisiológico provocado pela prática de uma sessão mais prolongada de exercício físico.

Por outro lado, demais investigações não apontaram impacto da duração do exercício na magnitude ou duração da HPE em sujeitos com PA normal( ${ }^{(30)}$ e elevada ${ }^{(5,30)}$. Assim, não é possível afirmar de forma definitiva se a duração de uma sessão de exercício físico modula a magnitude e a duração da HPE. Contudo, dados recentes mostram que a relação intensidade vs duração parece ser mais determinante na HPE de normotensos do que a ação isolada de tais variáveis ${ }^{(4)}$. Ou seja, uma sessão de exercício com menor intensidade e longa duração poderia ocasionar os mesmos resultados de HPE que uma sessão de alta intensidade e curta duração. Esse fato pode possibilitar a aplicação do exercício independentemente do estado clínico do sujeito, uma vez que hipertensos devem treinar com intensidade controlada.

\section{A INFLUÊNCIA DO TIPO DO EXERCÍCIO}

A massa muscular total envolvida na prática de um determinado exercício físico pode acarretar respostas metabólicas diferenciadas. Assim, exercícios físicos que envolvem grandes grupamentos musculares podem gerar maiores concentrações de íons e metabólitos que aparentemente estão relacionados com a HPE, como adenosina e potássio. Dessa forma, a HPE tem sido observada após a prática de uma sessão de variados tipos de exercícios aeróbios com certa diferença entre a massa muscular envolvida, como caminhada ${ }^{(9,28,42)}$, corrida ${ }^{(43)}$, ergômetro de pernas $^{(4,5,14,26,30,35,44)}$ e ergômetro de braço ${ }^{(45)}$. Ressalta-se que dos 53 estudos componentes dessa revisão, 37 utilizaram o cicloergômetro, 12 a esteira ergométrica, um a pista de atletismo, um o ergômetro de braço, um a corrida aquática e um a caminhada.

No entanto, são raros os estudos que buscaram comparar diretamente o efeito de diferentes tipos de exercício aeróbio no comportamento da HPE. MacDonald et al. ${ }^{(45)}$, por exemplo, concluíram que 30 min de exercício em maior massa muscular (cicloergômetro) não alteram a magnitude da HPE, mas podem proporcionar maior duração que o exercício de menor massa muscular (ergômetro de braço).
Em relação aos exercícios com discretas diferenças entre a massa muscular (cicloergômetro, caminhada e corrida), aparentemente não há inferência sobre o comportamento da HPE. Essa conclusão pode ser extrapolada, inclusive, para atividades no meio líquido. Dessa forma, Pontes et al. ${ }^{(46)}$ verificaram que a corrida aquática proporcionou HPE em hipertensos de forma semelhante ao exercício no meio seco. Porém, a forma de execução da atividade pode influenciar na HPE. Por exemplo, Park et al. ${ }^{(18)}$ demonstraram que o exercício intermitente proporciona maior HPE que o executado de forma contínua. Contudo, embora promissores, os dados de Pontes et al. ${ }^{(46)}$ e Park et al. ${ }^{(18)}$ necessitam de corroboração científica.

Com base nessas informações, verifica-se que o impacto do tipo de exercício na resposta hipotensora carece de maiores esclarecimentos, uma vez que os estudos disponíveis envolvendo essa temática são escassos, impedindo o estabelecimento de uma conclusão consistente sobre essa relação.

\section{A INFLUÊNCIA DO CICLO CIRCADIANO}

Em muitos indivíduos, a PA de repouso demonstra variação circadiana, caracterizada por queda dos valores pressóricos durante o período noturno e por aumento durante as horas após o despertar ${ }^{(47)}$. Os mecanismos responsáveis por essa variação podem relacionar-se ao ritmo circadiano endógeno, ao efeito estimulante do despertar e à ativação do sistema nervoso simpático(48).

Alguns pesquisadores demonstraram que a realização de exercício no período da tarde pode potencializar a magnitude da HPE durante o sono quando comparada com a de indivíduos que possuíam a queda natural da PA em função de ser sensíveis ao ciclo circadiano ${ }^{(15)}$. Jones et al. ${ }^{(31)}$ verificaram que durante o período da manhã a PA estava mais baixa em relação ao período da tarde. No entanto, esses mesmos pesquisadores somente detectaram a HPE na sessão realizada no período da tarde. Apesar desse indicativo da relação entre HPE e o ciclo circadiano, ainda são escassas as informações sobre o impacto do horário da realização da sessão de exercício e o comportamento da PA. Além disso, muitos pesquisadores não relataram o período do dia em que os protocolos de exercício foram realizados, o que impossibilita maiores inferência nesse aspecto.

\section{A INFLUÊNCIA DO TIPO DE POPULAÇÃO}

\section{Estado clínico}

A HPE tem sido documentada em indivíduos pré-hipertensos $(5,16,30,49)$ e hipertensos $(8,42,43,46,50)$. Por outro lado, apesar de não existir total consenso na literatura, vários estudos recentes com indivíduos normotensos identificaram queda na PA após exercícios de moderada intensidade, com redução da PA na ordem de $5-10 \mathrm{mmHg}^{(4,26,35,51,52)}$. Entretanto, a duração e a magnitude da HPE em normotensos parece ser menor que em hipertensos ${ }^{(20,21)}$. Isso pode ser explicado pelo fato de o débito cardíaco em indivíduos normotensos estar elevado durante o período da HPE, enquanto que em hipertensos o débito cardíaco deve diminuir(53). Pode-se especular que essa diferença entre hipertensos e normotensos relacione-se ao fato de que indivíduos hipertensos apresentem, em geral, deficiência em moduladores da resistência vascular periférica. Assim, como mecanismo de compensação, o sistema nervoso autônomo atuaria em função de reduzir a pressão arterial via débito cardíaco. Outro ponto interessante é que vários estudos verificaram redução da resistência periférica total no momento pós-exercício em normotensos ${ }^{(54,55)} \mathrm{e}$ também hipertensos ${ }^{(56)}$. No entanto, Hagberg et al. ${ }^{(50)}$, estudando idosos hipertensos, verificaram aumento da resistência periférica total após a sessão de exercício, sugerindo que o mecanismo ativado para a HPE pode estar relacionado com o estado clínico individual. 


\section{Etnia}

De todos os grupos étnicos, a prevalência de hipertensão arterial é mais comumente identificada em indivíduos negros ${ }^{(57)}$. As prováveis razões para esses achados podem relacionar-se com fatores como respostas hemodinâmicas e neuro-hormonais ao estresse ${ }^{(58)}$, alterações na sensitividade salina ${ }^{(59)}$, anormalidades na reatividade vascular(60), maior prevalência de sobrepeso e maior grau de inatividade física devido a interações genéticas e ambientais ${ }^{(61)}$ que restam ser identificados.

Os poucos estudos que buscaram identificar o impacto das respostas hipotensivas agudas em indivíduos negros ainda não são capazes de trazer uma clara evidência no que tange às possíveis diferenças raciais. Nesse sentido, Pescatello et al. ${ }^{(12)}$ verificaram, em mulheres brancas e negras com PA normal e elevada, que o exercício aeróbio pode provocar respostas diferenciadas entre as raças, uma vez que os níveis de PA das mulheres negras com PA elevada não se reduziram após o exercício aeróbio com duração de 40 min. Mesmo com poucos experimentos diretamente relacionados com a étnica, outros resultados corroboram o fato de que a resposta pós-exercício da PA pode ser diferente entre brancos e negros ${ }^{(62)}$

\section{Sexo}

Até o presente momento, é possível inferir que diferenças em relação ao sexo aparentemente não se relacionam com a HPE. Alguns estudos levantaram informações referentes ao comportamento da $\mathrm{PA}^{(63)}$ e da atividade neural simpática(64) em homens e mulheres. Esses estudos concluíram que PA e atividade neural simpática não guardam relação direta com o sexo. Além disso, tanto estudos que tiveram em suas amostras indivíduos de um sexo específico ${ }^{(65,66)}$ quanto aqueles cujas amostras eram compostas por homens e mulheres ${ }^{(13,54,67,68)}$ encontraram respostas hipotensoras similares. Porém, não foram encontrados experimentos que utilizaram mulheres em diferentes fases do ciclo menstrual, impedindo uma comparação entre respostas fisiológicas hormonais naturais do sexo feminino e HPE. Dessa forma, não há razão fisiológica para supor que o comportamento da HPE seja diferenciado entre os sexos.

\section{Faixa etária}

A HPE pode ocorrer independentemente da idade. Assim, estudos observaram HPE indivíduos jovens ${ }^{(69)}$, de meia-idade ${ }^{(69)}$ e idosos ${ }^{(50)}$. Destaca-se que intensidade e duração dos exercícios empregados nos delineamentos de pesquisa têm sido relativamente semelhantes em todas as faixas etárias. Em relação aos mecanismos, é importante salientar que, com o avançar da idade, há tendência natural para o aumento da resistência vascular periférica, uma vez que os vasos sanguíneos sofrem uma série de modificações estruturais, arquitetônicas e em sua composição ${ }^{(70)}$. Dessa forma, a HPE em idosos parece depender prioritariamente da queda do débito cardíaco, enquanto que em populações mais jovens a HPE tem sido atribuída à redução da resistência periférica total.

\section{Estado de treinamento}

O principal mecanismo postulado em relação à ocorrência da HPE em sedentários é a queda da resistência vascular periférica(23,25,71). Por outro lado, também é conhecido que o treinamento físico é associado a uma mudança na capacidade vasodilatadora ${ }^{(72)}$ e na regulação da $P A^{(73)}$, o que, por consequência, poderia inibir a queda pressórica em indivíduos treinados fisicamente. No entanto, diversos estudos envolvendo modelos animais e humanos demonstraram que a HPE pode ocorrer em população treinada ${ }^{(7,77)}$. Mais ainda, a magnitude da HPE não é diferente entre indivíduos treinados e aqueles classificados como sedentários ${ }^{(74)}$.
Por exemplo, Senitko et al. ${ }^{(74)}$ estudaram sujeitos de ambos os sexos ativos e inativos fisicamente, sendo verificada a ocorrência da HPE em todos os grupos. De maneira esperada, os indivíduos sedentários e as mulheres ativas apresentaram HPE devido a redução na resistência vascular periférica. Surpreendentemente, os homens ativos apresentaram queda no débito cardíaco, sendo que a resistência vascular periférica não alterou de maneira significativa. Posteriormente, Dujić et a ${ }^{(75)}$ investigaram a PA após um exercício máximo de curta duração em homens futebolistas profissionais. Esses autores, de maneira similar aos outros estudos, identificam HPE após a realização de uma única sessão de exercício físico e que o volume de ejeção e a capacidade pulmonar de difusão do monóxido de carbono nos pulmões se reduzem, enquanto a frequência cardíaca se eleva. Assim, com base nos estudos disponíveis, é possível verificar indícios de que a HPE ocorre similarmente em indivíduos treinados e sedentários. No entanto, os mecanismos responsáveis pela ocorrência desse fenômeno podem ser diferentes.

\section{MECANISMOS FISIOLÓGICOS}

O exato mecanismo responsável pela HPE ainda permanece desconhecido. No entanto, é possível inferir que sua ocorrência esteja relacionada com um conjunto de fatores que exerceriam influência em dois componentes fisiológicos: a resistência vascular periférica e o débito cardíaco. Vários estudos identificaram que a atividade nervosa simpática é inibida durante a HPE em humanos e em modelos animais, o que favorece a redução da resistência vascular periférica e, consequentemente, reduz os valores pressóricos ${ }^{(49,71)}$. Sabe-se ainda que, durante a elevação da PA, nervos aferentes que compõem o sistema barorreflexo são estimulados projetando-se no núcleo trato solitário, desencadeando bradicardia reflexa e vasodilatação periférica. De maneira inversa, durante a queda da PA, a estimulação dessas aferências junto ao núcleo trato solitário diminui, provocando taquicardia reflexa e vasoconstrição periférica na tentativa de normalizar a $\mathrm{PA}^{(76)}$. Outros possíveis mecanismos que podem relacionar-se com o aumento da inibição simpática pós-exercício são os barorreceptores cardiopulmonares. Um estudo ${ }^{(77)}$ utilizando modelos animais identificou que a HPE foi revertida após o bloqueio das aferências cardiopulmonares, sugerindo que os barorreceptores cardiopulmonares exercem grande influência na inibição simpática, uma vez que aparentemente são agentes que contribuem para manutenção da homeostase do sistema cardiovascular.

Recentemente, Mattace-Raso et al. ${ }^{(78)}$ verificaram que a resistência vascular foi positivamente associada com a sensitividade barorreflexa cardiovagal. Em modelos animais, Mousa et al. ${ }^{(79)}$ verificaram que a redução na atividade simpática e o aumento na função barorreflexa após o exercício são devidos a uma concomitante redução da angiotensina-II e dos receptores de angiotensina para o sistema nervoso central. É importante comentar que a angiotensina-Il atua no controle da PA interagindo com receptores na membrana celular das células-alvo (receptores AT1 e AT2). Por outro lado, diferentes comportamentos em relação à renina são identificados na literatura, de modo que alguns pesquisadores não verificaram alterações ${ }^{(80)}$ e outros identificaram aumentos nas concentrações circulantes ${ }^{(65,81)}$. Além disso, foram identificados aumentos nas concentrações de angiotensina-||(80).

Cabe destacar, ainda, que as catecolaminas apresentam direta relação com a resistência periférica, de modo que, durante o exercício, a medula adrenal é estimulada pelo sistema nervoso simpático, causando a liberação de adrenalina e noradrenalina em proporção à intensidade do exercício. A noradrenalina atua predominantemente nos receptores alfa-adrenérgicos periféricos causando vasoconstrição. 
A adrenalina, por sua vez, atua nos receptores vasculares beta-adrenérgicos, desencadeando moderado efeito vasodilatador. No entanto, parece que as concentrações de adrenalina e noradrenalina durante a HPE são semelhantes às do momento pré-exercício(27,42). Com isso, é possível afirmar que as catecolaminas aparentemente exercem pouca influência na HPE. O outro possível mecanismo da HPE relaciona-se com a liberação de adenosina pelos tecidos ativos durante o exercício. Alguns pesquisadores ${ }^{(44)}$, após utilizar a cafeína como bloqueador de adenosina, identificaram queda na magnitude da HPE. Entretanto, poucos estudos têm mensurado as concentrações de adenosina durante a HPE e, por isso, as contribuições da adenosina para a hipotensão pós exercício devem ser vistas com cautela. Também tem sido sugerido que as prostaglandinas liberadas durante o exercício podem ser parcialmente responsáveis pela HPE(82), uma vez que possuem efeito vasodilatador. Porém, ainda são raros os estudos sobre o papel das prostaglandinas na HPE e alguns dados não identificaram modificações significantes em relação à concentração de prostaglandinas na HPE ${ }^{(26)}$.

Um dos mais comentados agentes associados à HPE é o óxido nítrico. Essa substância é um importante sinalizador intra e extracelular sintetizado pelas células endoteliais, que converte a guanil ciclase em guanosina monofosfato cíclico, culminando com o relaxamento do músculo liso e, consequentemente, com a redução na resistência vascular periférica. Estudos conduzidos com modelos animais ${ }^{(83)}$ demonstraram que o óxido nítrico contribui para a HPE. Por outro lado, outras investigações que analisaram a relação do óxido nítrico com a HPE em humanos ${ }^{(24,25)}$ não identificaram contribuição significativa desse sinalizador.

\section{IMPLICAÇÕES DA HIPOTENSÃO PÓS-EXERCÍCIO}

A utilização de métodos não farmacológicos para auxiliar o controle da pressão arterial é fundamental para a proposição de tratamentos mais efetivos do ponto de vista da preservação e recuperação da função biológica. Dessa forma, a HPE destaca-se como um possível agente colaborador de problemas relacionados com o sistema cardiovascular.
No entanto, para que a HPE tenha relevância clínica, é importante que sua magnitude seja significativa e que perdure por várias horas. Assim, é importante observar que poucos estudos conseguiram identificar queda dos níveis ambulatoriais da PA em normotensos ${ }^{(20,21)}$. Porém, em hipertensos, vários estudos demonstraram efeito significante na PA ambulatorial ${ }^{(7-19,84)}$, mesmo com administração de drogas anti-hipertensivas ${ }^{(84)}$. Para além desses resultados promissores em hipertensos, é importante citar a possibilidade de riscos envolvidos na realização de exercícios físicos por indivíduos com limitações cardiovasculares. Assim, a tomada de medidas que visem o acompanhamento do comportamento das funções hemodinâmicas durante a realização das sessões de treinamento por pessoas portadoras de disfunções cardiovasculares é de grande importância para a manutenção da segurança dos procedimentos envolvendo exercício físico.

\section{CONCLUSÃO}

Apesar de serem encontrados diversos estudos que buscaram analisar o comportamento PA após a prática de uma sessão de exercícios aeróbios, ainda não há evidências em relação a muitos de seus mecanismos fisiológicos. Esse quadro pode ser compreendido na medida em que PA é uma variável influenciada por diversos fatores e a contribuição efetiva de cada mecanismo ainda não está esclarecida. Além disso, diferenças em relação aos protocolos de exercício e das amostras investigadas enfraquecem as conclusões. Nesse sentido, a influência da intensidade, duração e tipo de exercício permanece de certa forma obscura. No entanto, a maioria dos estudos que verificaram a ocorrência da HPE tem utilizado protocolos de exercício com duração de 15 a 60 min e intensidade em torno de $60 \%$ do $V_{2 \text { pico. }}$ Por outro lado, o estado clínico parece estar relacionado com a resposta hipotensora pós-exercício, pois indivíduos hipertensos têm apresentado maior tempo e magnitude de queda da PA pós-exercício em relação aos normotensos.

$\overline{\text { Todos os autores declararam não haver qualquer potencial conflito de }}$ interesses referente a este artigo.

\section{REFERÊNCIAS BIBLIOGRÁFICAS}

1. Kearney PM, Whelton M, Reynolds K, Whelton PK, He J. Worldwide prevalence of hypertension: a systematic review. J Hypertens. 2004;22(1):11-9. Review.

2. Pescatello LS, Franklin BA, Fagard R, Farquhar WB, Kelley GA, Ray CA; American College of Sports Medicine. American College of Sports Medicine position stand. Exercise and hypertension. Med Sci Sports Exerc. 2004;36(3):533-53. Review.

3. Cornelissen VA, Fagard RH. Effects of endurance training on blood pressure, blood pressure-regulating mechanisms, and cardiovascular risk factors. Hypertension. 2005;46(4):667-75.

4. Jones $\mathrm{H}$, George K, Edwards B, Atkinson G. Is the magnitude of acute post-exercise hypotension mediated by exercise intensity or total work done? Eur J Appl Physiol. 2007;102(1):33-40.

5. Guidry MA, Blanchard BE, Thompson PD, Maresh CM, Seip RL, Taylor AL, et al. The influence of short and long duration on the blood pressure response to an acute bout of dynamic exercise. Am Heart J. 2006;151(6):1322.e5-12.

6. Hill L. Arterial pressure in man while sleeping, resting, working and bathing. J Physiol Lond. 1897;22(xxvi-xxix).

7. Pescatello LS, Fargo AE, Leach CN Jr, Scherzer HH. Short-term effect of dynamic exercise on arterial blood pressure. Circulation. 1991;83(5):1557-61.

8. Pescatello LS, Miller B, Danias PG, Werner M, Hess M, Baker C, et al. Dynamic exercise normalizes resting blood pressure in mildly hypertensive premenopausal women. Am Heart J. 1999;138(5 Pt 1):916-21.

9. Wallace JP, Bogle PG, King BA, Krasnoff JB, Jastremski CA. The magnitude and duration of ambulatory blood pressure reduction following acute exercise. J Hum Hypertens. 1999;13(6):361-6.

10. Quinn TJ. Twenty-four hour, ambulatory blood pressure responses following acute exercise: impact of exercise intensity. J Hum Hypertens. 2000;14(9):547-53.
11. Pescatello LS, Kulikowich JM. The aftereffects of dynamic exercise on ambulatory blood pressure. Med Sci Sports Exerc. 2001;33(11):1855-61. Review.

12. Pescatello LS, Bairos L, Vanheest JL, Maresh CM, Rodriguez NR, Moyna NM, et al. Postexercise hypotension differs between white and black women. Am Heart J. 2003;145(2):364-70.

13. Taylor-Tolbert NS, Dengel DR, Brown MD, McCole SD, Pratley RE, Ferrell RE, et al. Ambulatory blood pressure after acute exercise in older men with essential hypertension. Am J Hypertens. 2000;13(1 Pt 1):44-51

14. Pescatello LS, Guidry MA, Blanchard BE, Kerr A, Taylor AL, Johnson AN, et al. Exercise intensity alters postexercise hypotension. J Hypertens. 2004;22(10):1881-8.

15. Park S, Jastremski CA, Wallace JP. Time of day for exercise on blood pressure reduction in dipping and nondipping hypertension. J Hum Hypertens. 2005;19(8):597-605.

16. Syme AN, Blanchard BE, Guidry MA, Taylor AW, Vanheest JL, Hasson S, et al. Peak systolic blood pressure on a graded maximal exercise test and the blood pressure response to an acute bout of submaximal exercise. Am J Cardiol. 2006;98(7):938-43.

17. Blanchard BE, Tsongalis GJ, Guidry MA, LaBelle LA, Poulin M, Taylor AL, et al. RAAS polymorphisms alter the acute blood pressure response to aerobic exercise among men with hypertension. Eur J Appl Physiol. 2006;97(1):26-33

18. Park S, Rink LD, Wallace JP. Accumulation of physical activity leads to a greater blood pressure reduction than a single continuous session, in prehypertension. J Hypertens. 2006;24(9):1761-70.

19. Pescatello LS, Turner D, Rodriguez N, Blanchard BE, Tsongalis GJ, Maresh CM, et al. Dietary calcium intake and renin angiotensin system polymorphisms alter the blood pressure response to aerobic exercise: a randomized control design. Nutr Metab (Lond). 2007;4:1. 
20. Bermudes AM, Vassallo DV, Vasquez EC, Lima EG. Ambulatory blood pressure monitoring in normotensive individuals undergoing two single exercise sessions: resistive exercise training and aerobic exercise training. Arq Bras Cardiol. 2004;82(1):65-71, 57-64.

21. Forjaz CL, Tinucci T, Ortega KC, Santaella DF, Mion D Jr, Negrão CE. Factors affecting post-exercise hypotension in normotensive and hypertensive humans. Blood Press Monit. 2000;5(5-6):255-62.

22. Brownley KA, West SG, Hinderliter AL, Light KC. Acute aerobic exercise reduces ambulatory blood pressure in borderline hypertensive men and women. Am J Hypertens. 1996;9(3):200-6.

23. Halliwill JR, Taylor JA, Hartwig TD, Eckberg DL. Augmented baroreflex heart rate gain after moderateintensity, dynamic exercise. Am J Physiol. 1996;270(2 Pt 2):R420-6.

24. Sharman JE, McEniery CM, Campbell R, Pusalkar P, Wilkinson IB, Coombes JS, et al. Nitric oxide does not significantly contribute to changes in pulse pressure amplification during light aerobic exercise. Hypertension. 2008;51(4):856-61.

25. Halliwill JR, Minson CT, Joyner MJ. Effect of systemic nitric oxide synthase inhibition on postexercise hypotension in humans. J Appl Physiol. 2000;89(5):1830-6.

26. Lockwood JM, Pricher MP, Wilkins BW, Holowatz LA, Halliwill JR. Postexercise hypotension is not explained by a prostaglandin-dependent peripheral vasodilation. J Appl Physiol. 2005;98(2):447-53.

27. MacDonald JR, Rosenfeld JM, Tarnopolsky MA, Hogben CD, Ballantyne CS, MacDougall JD. Post exercise hypotension is not mediated by the serotonergic system in borderline hypertensive individuals. Hum Hypertens. 2002;16(1):33-9.

28. Wallace JP, Bogle PG, King BA, Krasnoff JB, Jastremski CA. A comparison of 24-h average blood pressures and blood pressure load following exercise. Am J Hypertens. 1997;10(7 Pt 1):728-34.

29. Forjaz CL, Santaella DF, Rezende LO, Barretto AC, Negrão CE. [Effect of exercise duration on the magnitude and duration of post-exercise hypotension]. Arq Bras Cardiol. 1998;70(2):99-104. Portuguese.

30. MacDonald JR, MacDougall JD, Hogben CD. The effects of exercise duration on post-exercise hypotension. J Hum Hypertens. 2000;14(2):125-9.

31. Jones H, Pritchard C, George K, Edwards B, Atkinson G. The acute post-exercise response of blood pressure varies with time of day. Eur J Appl Physiol. 2008;104(3):481-9.

32. MacDonald JR, Hogben CD, Tarnopolsky MA, MacDougall JD. Post exercise hypotension is sustained during subsequent bouts of mild exercise and simulated activities of daily living. J Hum Hypertens. 2001;15(8):567-71.

33. Forjaz CL, Matsudaira Y, Rodrigues FB, Nunes N, Negrão CE. Post-exercise changes in blood pressure, heart rate and rate pressure product at different exercise intensities in normotensive humans. Braz $J$ Med Biol Res. 1998;31(10):1247-55.

34. MacDonald J, MacDougall J, Hogben C. The effects of exercise intensity on post exercise hypotension J Hum Hypertens. 1999;13(8):527-31

35. Forjaz CL, Cardoso CG Jr, Rezk CC, Santaella DF, Tinucci T. Postexercise hypotension and hemodynamics: the role of exercise intensity. J Sports Med Phys Fitness. 2004;44(1):54-62.

36. Alderman BL, Arent SM, Landers DM, Rogers TJ. Aerobic exercise intensity and time of stressor administration influence cardiovascular responses to psychological stress. Psychophysiology. 2007:44(5):759-66

37. Piepoli M, Isea JE, Pannarale G, Adamopoulos S, Sleight P, Coats AJ. Load dependence of changes in forearm and peripheral vascular resistance after acute leg exercise in man. J Physiol. 1994;478(Pt 2):357-62

38. Seals DR, Rogers MA, Hagberg JM, Yamamoto C, Cryer PE, Ehsani AA. Left ventricular dysfunction after prolonged strenuous exercise in healthy subjects. Am J Cardiol. 1988;61(11):875-9.

39. Bennett T, Wilcox RG, Macdonald IA. Post-exercise reduction of blood pressure in hypertensive men is not due to acute impairment of baroreflex function. Clin Sci (Lond). 1984;67(1):97-103.

40. Mach C, Foster C, Brice G, Mikat RP, Porcari JP. Effect of exercise duration on postexercise hypotension. J Cardiopulm Rehabil. 2005;25(6):366-9.

41. Overton JM, Joyner MJ, Tipton CM. Reductions in blood pressure after acute exercise by hypertensive rats. J Appl Physiol. 1988;64(2):748-52.

42. Wilcox RG, Bennett T, Macdonald IA, Broughton Pipkin F, Baylis PH. Post-exercise hypotension: the effects of epanolol or atenolol on some hormonal and cardiovascular variables in hypertensive men Br J Clin Pharmacol. 1987;24(2):151-62.

43. Rueckert PA, Slane PR, Lillis DL, Hanson P. Hemodynamic patterns and duration of post-dynamic exercise hypotension in hypertensive humans. Med Sci Sports Exerc. 1996;28(1):24-32.

44. Notarius CF, Morris BL, Floras JS. Caffeine attenuates early post-exercise hypotension in middle-aged subjects. Am J Hypertens. 2006;19(2):184-8.

45. MacDonald JR, MacDougall JD, Hogben CD. The effects of exercising muscle mass on post exercise hypotension. J Hum Hypertens 2000;14(5):317-20.

46. Pontes FL Jr, Bacurau RF, Moraes MR, Navarro F, Casarini DE, Pesquero JL, et al. Kallikrein kinin system activation in post-exercise hypotension in water running of hypertensive volunteers. Int Immunopharmacol. 2008;8(2):261-6.

47. Kaplan NM. Morning surge in blood pressure. Circulation. 2003;107(10):1347.

48. Khoury AF, Sunderajan P, Kaplan NM. The early morning rise in blood pressure is related mainly to ambulation. Am J Hypertens. 1992;5(6 Pt 1):339-44.

49. Floras JS, Sinkey CA, Aylward PE, Seals DR, Thoren PN, Mark AL. Postexercise hypotension and sympathoinhibition in borderline hypertensive men. Hypertension. 1989;14(1):28-35.

50. Hagberg JM, Montain SJ, Martin WH 3rd. Blood pressure and hemodynamic responses after exercise in older hypertensives. J Appl Physiol. 1987;63(1):270-6.

51. Moraes MR, Bacurau RF, Ramalho JD, Reis FC, Casarini DE, Chagas JR, et al. Increase in kinins on postexercise hypotension in normotensive and hypertensive volunteers. Biol Chem. 2007;388(5):533-40.

52. Harvey PJ, Morris BL, Kubo T, Picton PE, Su WS, Notarius CF, et al. Hemodynamic after-effects of acute dynamic exercise in sedentary normotensive postmenopausal women. J Hypertens. 2005;23(2):285-92

53. Floras JS, Wesche J. Haemodynamic contributions to post-exercise hypotension in young adults with hypertension and rapid resting heart rates. J Hum Hypertens. 1992;6(4):265-9.
54. Coats AJ, Conway J, Isea JE, Pannarale G, Sleight P, Somers VK. Systemic and forearm vascular resistance changes after upright bicycle exercise in man. J Physiol. 1989:413:289-98.

55. Isea JE, Piepoli M, Adamopoulos S, Pannarale G, Sleight P, Coats AJ. Time course of hemodynamic changes after maximal exercise. Eur J Clin Invest. 1994;24(12):824-9.

56. Cléroux J, Kouamé N, Nadeau A, Coulombe D, Lacourcière Y. Aftereffects of exercise on regional and systemic hemodynamics in hypertension. Hypertension. 1992;19(2):183-91.

57. Rosamond W, Flegal K, Furie K, Go A, Greenlund K, Haase N, et al. Heart disease and stroke statistics 2008 update: a report from the American Heart Association Statistics Committee and Stroke Statistics Subcommittee. Circulation. 2008;117(4):e25-146.

58. Cardillo C, Kilcoyne CM, Cannon RO 3rd, Panza JA. Racial differences in nitric oxide-mediated vasodilator response to mental stress in the forearm circulation. Hypertension. 1998;31(6):1235-9.

59. Svetkey LP, Chen YT, McKeown SP, Preis L, Wilson AF. Preliminary evidence of linkage of salt sensitivity in black Americans at the beta 2-adrenergic receptor locus. Hypertension. 1997;29(4):918-22.

60. Gokce N, Holbrook M, Duffy SJ, Demissie S, Cupples LA, Biegelsen E, et al. Effects of race and hypertension on flow-mediated and nitroglycerin-mediated dilation of the brachial artery. Hypertension. 2001;38(6):1349-54.

61. Abate NI, Mansour YH, Tuncel M, Arbique D, Chavoshan B, Kizilbash A, et al. Overweight and sympathetic overactivity in black Americans. Hypertension. 2001;38(3):379-83.

62. Headley SA, Keenan TG, Manos TM, Phillips K, LachowetzT, Keenan HA, et al. Renin and hemodynamic responses to exercise in borderline hypertensives. Ethn Dis. 1998;8(3):312-8.

63. Hayward CS, Kelly RP. Gender-related differences in the central arterial pressure waveform. J Am Coll Cardiol. 1997;30(7):1863-71.

64. Matsukawa T, Sugiyama Y, Watanabe T, Kobayashi F, Mano T. Gender difference in age-related changes in muscle sympathetic nerve activity in healthy subjects. Am J Physiol. 1998;275(5 Pt 2):R1600-4.

65. Paulev PE, Jordal R, Kristensen O, Ladefoged J. Therapeutic effect of exercise on hypertension. Eur J Appl Physiol Occup Physiol. 1984;53(2):180-5.

66. Headley SA, Claiborne JM, Lottes CR, Korba CG. Hemodynamic responses associated with post-exercise hypotension in normotensive black males. Ethn Dis. 1996;6(1-2):190-201.

67. Raglin JS, Turner PE, Eksten F. State anxiety and blood pressure following 30 min of leg ergometry or weight training. Med Sci Sports Exerc. 1993;25(9):1044-8

68. Brown SP, Clemons JM, He Q, Liu S. Effects of resistance exercise and cycling on recovery blood pressure. J Sports Sci. 1994;12(5):463-8.

69. Kaufman FL, Hughson RL, Schaman JP. Effect of exercise on recovery blood pressure in normotensive and hypertensive subjects. Med Sci Sports Exerc. 1987;19(1):17-20.

70. Bilato C, Crow MT. Atherosclerosis and the vascular biology of aging. Aging (Milano). 1996;8(4):221-34.

71. Halliwill JR, Taylor JA, Eckberg DL. Impaired sympathetic vascular regulation in humans after acute dynamic exercise. J Physiol. 1996;495(Pt 1):279-88.

72. Martin WH 3rd, Ogawa T, Kohrt WM, Malley MT, Korte E, Kieffer PS, et al. Effects of aging, gender, and physical training on peripheral vascular function. Circulation. 1991;84(2):654-64.

73. Raven PB, Pawelczyk JA. Chronic endurance exercise training: a condition of inadequate blood pressure regulation and reduced tolerance to LBNP. Med Sci Sports Exerc. 1993;25(6):713-21. Review.

74. Senitko AN, Charkoudian N, Halliwill JR. Influence of endurance exercise training status and gender on postexercise hypotension. J Appl Physiol. 2002;92(6):2368-74.

75. Dujic Z, Ivancev V, Valic Z, Bakovic D, Marinovic-Terzic I, Eterovic D, et al. Postexercise hypotension in moderately trained athletes after maximal exercise. Med Sci Sports Exerc. 2006;38(2):318-22.

76. Krieger EM, Brum PC, Negrão CE. Role of arterial baroreceptor function on cardiovascular adjustments to acute and chronic dynamic exercise. Biol Res. 1998;31(3):273-9. Review.

77. Collins HL, DiCarlo SE. Attenuation of postexertional hypotension by cardiac afferent blockade. Am J Physiol. 1993;265(4 Pt 2):H1179-83.

78. Mattace-Raso FU, van den Meiracker AH, Bos WJ, van der Cammen TJ, Westerhof BE, Elias-Smale S, et al. Arterial stiffness, cardiovagal baroreflex sensitivity and postural blood pressure changes in older adults: the Rotterdam Study. J Hypertens. 2007;25(7):1421-6.

79. Mousa TM, Liu D, Cornish KG, Zucker IH. Exercise training enhances baroreflex sensitivity by an angiotensin II-dependent mechanism in chronic heart failure. J Appl Physiol. 2008;104(3):616-24.

80. Wilcox RG, Bennett T, Brown AM, MacDonald IA. Is exercise good for high blood pressure? Br Med J (Clin Res Ed). 1982;285(6344):767-9.

81. Piepoli M, Coats AJ, Adamopoulos S, Bernardi L, Feng YH, Conway J, et al. Persistent peripheral vasodilation and sympathetic activity in hypotension after maximal exercise. J Appl Physiol. 1993;75(4):1807-14.

82. Ward ME. Dilation of rat diaphragmatic arterioles by flow and hypoxia: roles of nitric oxide and prostaglandins. J Appl Physiol. 1999;86(5):1644-50.

83. Rao SP, Collins HL, DiCarlo SE. Postexercise al pha-adrenergic receptor hyporesponsiveness in hypertensive rats is due to nitric oxide. Am J Physiol Regul Integr Comp Physiol. 2002;282(4):R960-8.

84. Ciolac EG, Guimarães GV, D Àvila VM, Bortolotto LA, Doria EL, Bocchi EA. Acute effects of continuous and interval aerobic exercise on 24-h ambulatory blood pressure in long-term treated hypertensive patients. Int J Cardiol. 2008; in press.

85. MacDonald JR, MacDougall JD, Interisano SA, Smith KM, McCartney N, Moroz JS et al. Hypotension following mild bouts of resistance exercise and submaximal dynamic exercise. Eur J Appl Physiol Occup Physiol. 1999;79(2):148-54.

86. Birch K, Cable N, George K. Combined oral contraceptives do not influence post-exercise hypotension in women. Exp Physiol. 2002;87(5):623-32.

87. Terziotti P, Schena F, Gulli G, Cevese A. Post-exercise recovery of autonomic cardiovascular control: a study by spectrum and cross-spectrum analysis in humans. Eur J Appl Physiol. 2001;84(3):187-94.

88. Brandão Rondon MU, Alves MJ, Braga AM, Teixeira OT, Barretto AC, Krieger EM, et al. Postexercise blood pressure reduction in elderly hypertensive patients. J Am Coll Cardiol. 2002;39(4):676-82. 